\title{
Heuristic Algorithm for Minimizing the Electricity Cost of Smart House
}

\author{
Mohamed Arikiez ${ }^{1}$, Faisal Alotaibi ${ }^{1}$, Farouq Gdhaidh ${ }^{2}$, Radwan Khershif $^{3}$ and Salahedin Rehan ${ }^{4}$ \\ 1. Department of Computer Science, University of Liverpool, Liverpool L69 3BX, UK \\ 2. Michanical Engineering, Univesity of Bradford, Bradford BD7 1DP, UK \\ 3. Electrical and Electronic Department, Zawia University, Zawia Z1 14403, Libya \\ 4. Electrical and Electronic Department, University of York, York YO10 5DD, UK
}

Received: November 24, 2016 / Accepted: December 01, 2016 / Published: April 30, 2017.

\begin{abstract}
This framework proposes a heuristic algorithm based on LP (linear programming) for optimizing the electricity cost in large residential buildings, in a smart grid environment. Our heuristic tackles large multi-objective energy allocation problem (large number of appliances and high time resolution). The primary goal is to reduce the electricity bills, and discomfort factor. Also, increase the utilization of domestic renewable energy, and reduce the running time of the optimization algorithm. Our heuristic algorithm uses linear programming relaxation, and two rounding strategies. The first technique, called CR (cumulative rounding), is designed for thermostatic appliances such as air conditioners and electric heaters, and the second approach, called MCR (minimum cost rounding), is designed for other interruptible appliances. The results show that the proposed heuristic algorithm can be used to solve large MILP (mixed integer linear programming) problems and gives a decent suboptimal solution in polynomial time.
\end{abstract}

Key words: Smart grid, mixed integer linear programming, LP relaxation, demand side management, demand response, multi-objective optimization, heuristic allocation algorithm.

\section{Introduction}

Residential buildings consume around $48 \%$ of the available energy in the world. This significant growth of the global population is causing a serious issue in the power sector. As a result, electricity bills are increasing at a significant rate. The investment in building new power plants (traditional or renewable plants) would not cover the electricity demand in the future [1]. This situation can be improved in several ways. For instance, improving the generation side (increase generation capacity) using more renewable resources, building or/and developing more efficient and environmentally friendly power plants. Moreover, electricity bills can be reduced by using domestic renewable power, home automation techniques, smart

Corresponding author: Mohamed Arikiez, Ph.D., lecturer, research fields: optimization algorithm, smart grids, renewable power. appliances, and smart grid technology such as DSM (demand side management) and DR (demand response) to encourage consumers to modify their power demand.

The concept of the smart grid is relatively new. It is an enhanced electrical grid where information and communications technology can be used between the grid entities to improve the power system and increase the profit of consumers, distributors and generation companies. The key features of the smart grid are reliability, flexibility, efficiency, sustainability, peak curtailment, demand response, market enabling, platform for advanced services, manageability of the available resources. To exploit smart grid in residential buildings, we need a new technology in these buildings such as integrated communications, sensing and measurements, smart meters, advanced control, advanced components, and power generation [2]. 
There are two main kinds of optimization techniques, which are classical or exact, and heuristic. Many optimization techniques have been used to optimize the energy cost in the residential buildings, including, but not limited to, decision system, static, adaptive dynamic programming, evolutionary programming, and intelligent systems [3]. Game theory is a kind of decision system algorithms, Refs. $[4,5]$ use demand side management based on game theory in smart grid to reduce the cost. Besides, NN (neural networks) are a sort of intelligent systems, Matallanas et al. [6] and Argiriou et al. [7] use NN to find the optimal solution for a house load to optimize the cost of electricity. Moreover, PSO (particle swarm optimization) is evolutionary programming; Refs. [8-13] use this heuristic algorithm to optimize the load of household appliances. Additionally, Ref. [14] exploits a heuristic algorithm based on EA (evolutionary algorithms) to reduce the cost of power consumption. GA (genetic algorithms), also, are evolutionary algorithms. Frameworks [15, 16] investigate using GA as heuristic optimization algorithm in a residential building.

LP (linear programming), IP (integer programming), and MILP (mixed integer linear programming) are static optimization techniques (exact algorithms), and they are widely used in different fields. Many studies have exploited MILP to solve this problem [17-32]. Although these studies add a real contribution to the area, they have not tackled the computation time of the algorithm to solve the large problem efficiently. MILP is NP-hard problem which means the computation time could go to infinity if the number of variables in the model is large (large number of appliances). Ref. [33] considered waiting time for household appliances but not the computation time, whereas Ref. [24] introduced a technique to reduce the computation time, which is using a variant partition of time intervals. However, the computation time using a powerful computer is still significant (414.9 s), and this number could soar by increasing the number of appliances in the problem.

The relaxation technique is used in large and complicated problems to find a near-optimal solution. Lagrangian relaxation has been utilized in Refs. [34-36] to divide the optimization problem to a set of small problems to decrease the complexity of the problem, whereas LP relaxation is used in Ref. [37] to reduce the complexity of quadratic integer programming. LP relaxation works by omitting integer constraints on variables in IP/MILP.

The main contribution of this paper is that it uses a heuristic algorithm based on linear programming to solve the large multi-objective optimization problem in a polynomial time. Our heuristic algorithm reduces the number of integer variables in the problem by using LP relaxation and rounding technique to find a near-optimal solution so that it can solve large and complicated problems in a relatively short time. Two rounding techniques have been introduced, one for thermostatic appliances (electric heaters and AC units), called CR (cumulative rounding), and another rounding strategy for the other interruptible appliances, called MPR (maximum profit rounding). Also, empirical evaluation is presented and discussed in this paper.

The remainder of this paper is organized as follows. System modeling is presented in Section 2, whereas problem formulation is demonstrated in Section 3. After that, results and discussions are illustrated. Finally, we concluded our paper and listed the references.

\section{Problem Statement}

\subsection{System Definition}

In this section, we present the formalization of the computational problem discussed in this paper. Let us assume that we have a large house equipped with domestic renewable micro plant (photovoltaic array or wind turbine) that generates $P(t)$ Watt/time unit. The house has a set of smart appliances, $\mathcal{A}$. In it, the number of these appliances is $\mathrm{N} \equiv|\mathcal{A}|$. The 
house is connected to electricity smart grid (via smart meter) that provides the customer with electricity at rate $\lambda(t) / \mathrm{kWh}$. The electricity from local renewable micro plant can either be used immediately at the property (at a unit cost of $\xi(t)$ )/kWh, or exported to the NEG (National Electricity Grid) and the owner is awarded a monetary premium of $\zeta(t) £$ or $\$$ per $\mathrm{kWh}$. All appliances in the building are controlled by an energy manager, whose primary task is to minimize the electricity cost, keep the house temperature at comfortable level, and minimize the discomfort factor. Also, it enhances the utilization of renewable power. The allocated power to household appliances could be from the grid, local power, or both. See Fig. 1.

\subsection{Household Electric Appliances}

The smart house is equipped with a set of electric appliances $\mathcal{A}$, all household appliances are the main energy outlets. We assume that the appliances in the system can be easily switched ON or OFF via remote controller system. Washing machines, cookers, AC (air-conditioner) units, battery chargers are examples of suitable appliances, whereas TV sets or computers do not fit into such framework. Moreover, we assume that the appliances can either be interruptible or uninterruptible, uni-phase or multiphase. Interruptible appliances are designed to be switched ON/OFF at any time. Appliances of this type include heaters, cookers, or AC units. Uninterruptible appliances are not designed to be switched OFF once they have been switched ON until they finish a particular task. Washing machines are good examples of uninterruptible appliances. Electric heaters are, also, good example of uni-phase appliances. Any such appliance can either be OFF or $\mathrm{ON}$ and when it is $\mathrm{ON}$ it uses approximately a constant amount of power (nominal power). Fig. 2 shows the power profile of a set of household appliances.

To model the power profiles of household appliances, we need to do some approximation for all power

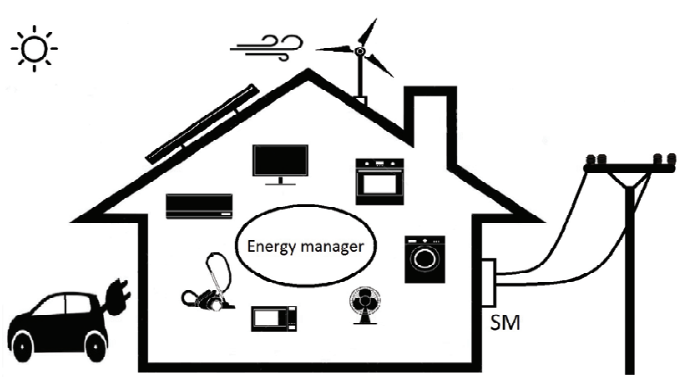

Fig. 1 Smart house, with large number of household appliances, connected to smart grid.

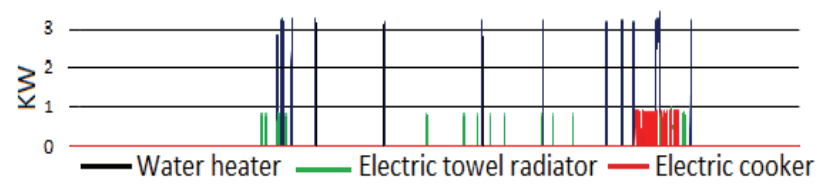

(a) Real data of power profile of a set of uniphase appliances (electric water heater, electric towel radiator, electric cooker)

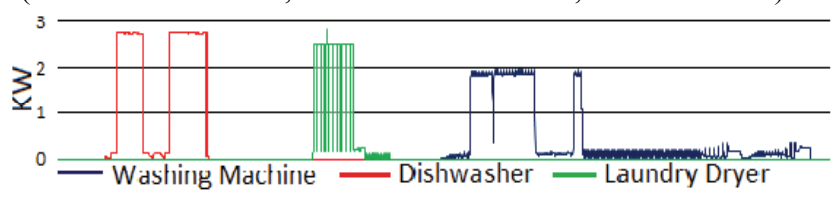

(b) Real data of power profile of set of multiphase appliances (washing machine, dishwasher, laundry dryer)

Fig. 2 Uniphase vs. multiphase appliances.

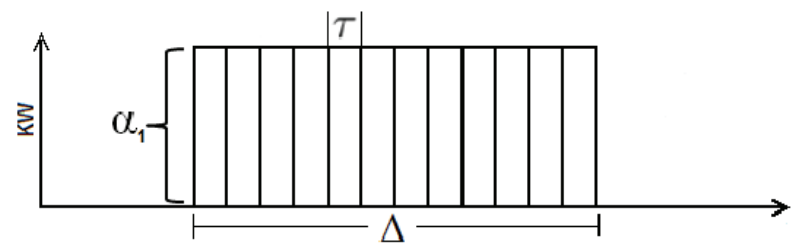

(a) Mathematical model of power profile of uniphase household appliance

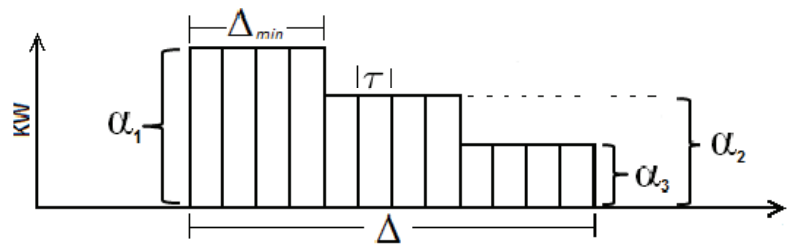

(b) Mathematical model of power profile of multiphase household appliance

Fig. 3 Uniphase vs. multiphase appliances.

profiles, see Fig. 3. Fig. 3a shows the power profile of a uniphase appliance, whereas Fig. $3 b$ illustrates the power profile of a multiphase appliance.

For the purpose of this study we assume that each appliance $\mathcal{A}$ operates in $\Delta>0$ (nominal) phases and for each appliance, it is possible to define a power profile vector $\left(\alpha_{1}, \ldots, \alpha_{\Delta}\right)$ describing its energy needs, see Fig. 4 . We assume that $\Delta_{\min }$ is the length of the 


\section{\begin{tabular}{|l|l|l|l|l|l|l|l|l|l|l|l|l|}
\hline$\alpha_{1}$ & $\alpha_{2}$ & $\alpha_{3}$ & $\cdots$ & $\cdots$ & & & & & & & & $\cdots$ \\
\hline
\end{tabular}}

Fig. 4 Power profile vector of household appliance.

shortest phase. Each $\alpha_{j}$ is a non-negative real number $\left(\alpha_{j} \geq 0\right)$, corresponding to the average amount of power used by the appliance during its $j$ th phase. When switched on, appliance $\mathcal{A}$ progresses through each of its phases, starting from phase 1 up until phase $\Delta$ at which point the appliance is switched OFF. We also assume that for each appliance we know whether it is interruptible or not, and the number of times must be used, $n$. Note that such a model fits the different types of appliances described before.

\subsection{Optimization Problem}

In this setting, a cost function $\Psi$ is associated with the building, the cost, in this case, combines three components (NEG electricity cost plus the cost of domestic renewable power minus the profit made by selling electricity to the grid). The cost function of the proposed mathematical model is presented in the following Eq. (1):

$$
\Psi=\int \lambda(t) L_{g}(t) d t+\int \xi(t) L_{r}(t) d t-\int \zeta(t) E(t) d t
$$

where, $L_{g}(t)$ describes the amount of allocated UG energy to the AC units, $L_{r}(t)$ is the amount of renewable power used in the building, and $E(t)$ the amount of renewable power exported to the NEG. Furthermore, each apartment, $r$, has a discomfort factor or function, $\Xi_{r}$, defined as follows:

$$
\Xi_{r}=\int\left|T_{i n}^{r}(t)-T_{o p t}^{r}\right| d t
$$

The discomfort factor for whole building is illustrated in the following Eq. (3),

$$
\Omega=\sum_{\forall r} \Xi_{r}
$$

The problem of allocating electrical energy to a set of household appliances in a way that satisfies a set of given constraints and is cost-effective for the users, $\Pi$, is equivalent to minimizing both functions, $\Psi$ and $\Omega$.

$$
\min (\Psi, \Omega) \text {, }
$$

subject to a set of constraints (user preferences, power, and temperature constraints).

\section{MILP Formulation}

The importance of combinatorial optimization comes from the fact that it can solve many problems. The computational problem defined in Section 2 lends itself naturally to a simple mathematical programming formulation, provided time is discretized and confined to a window of a finite width.

\subsection{Time Horizon}

From now on, we assume that each instance of the given problem is solved over a finite time window, and that the time horizon is subdivided into a finite number of time slots, $\mathcal{T}=\left\{t_{1}, t_{2}, \ldots, t_{\mathrm{T}}\right\}$, all of length $\mathcal{T}$ time unit, see Fig. 6.

\subsection{Multiphase Appliances}

Let us assume that a residential building has a set of multiphase appliances, $\dddot{\mathcal{A}} \subset \mathcal{A}, \mathrm{m}=|\dddot{\mathcal{A}}|$. We, also, assume that each appliance $i$ runs through $\Delta_{i}$ (real) phases, of length $\mathcal{T}$, and divided to a set of phases, called virtual appliances $\left(\mathcal{J}_{i}\right)$. In addition, we assume that real phases are grouped into clutches of length $\Delta_{\min }$ corresponding to the nominal phases and the appliances are uninterruptible within each clutch (Fig. 3b shows an appliance with three clutches). We use a dedicated binary decision variable $x_{j}^{i}(t)$ for each virtual appliance $j$ belonging to appliance $i$. The variable holds the appliance ON/OFF state at time slot $t$.

$$
\forall i \quad P_{j}^{i}(t)=\alpha_{j}^{i} \cdot x_{j}^{i}(t) \in\left\{0, \ldots, \alpha_{\Delta_{i}}\right\}, \forall j: j \in J_{i}
$$

We, also, assume that appliance $i$ can only be run between time slot $t_{s}^{i}$ and $t_{f}^{i}$ (with $t_{s}^{i} \leq t_{f}^{i}$ ), in a so-called comfort interval specified by the user, if needed. We model this using the following constraints,

$$
\forall i \quad \sum_{t=0}^{t_{s}^{i}} x_{j}^{i}(t)+\sum_{t=t_{f 1}^{i}+1}^{t_{T}} x_{j}^{i}(t)=0, \forall j: j \in J_{i}
$$




\section{\begin{tabular}{|l|l|l|l|l|l|l|l|l|l|l|l|l|l|l|}
\hline 3.1 & 3.1 & 3.1 & 3.1 & 3.1 & 3.1 & 3.1 & 3.1 & 3.1 & 3.1 & 3.1 & 3.1 & 3.1 & 3.1 & 3.1 \\
\hline
\end{tabular} \\ \begin{tabular}{|l|l|l|l|l|l|l|l|l|l|l|l|l|l|l|}
\hline 3.1 & 3.1 & 3.1 & 0.0 & 3.1 & 3.1 & 3.1 & 3.1 & 0.0 & 3.1 & 0.0 & 3.1 & 0.0 & 0.0 & 3.1 \\
\hline
\end{tabular}}

(a) Example, power profile vector of uni-phase appliance

\begin{tabular}{|l|l|l|l|l|l|l|l|l|l|l|l|l|l|l|}
\hline 3.1 & 3.1 & 3.1 & 3.1 & 0.2 & 0.2 & 0.2 & 3.1 & 3.1 & 3.1 & 3.1 & 3.1 & 0.2 & 0.2 & 0.2 \\
\hline 3.1 & 3.1 & 3.1 & 3.1 & 0.2 & 0.2 & 0.2 & 0.0 & 0.0 & 0.0 & 3.1 & 3.1 & 3.1 & 3.1 & 3.1 \\
\hline
\end{tabular}

(b) Example, power profile vector of multiphase appliance

Fig. 5 Example, power profile vector of uni-phase and multiphase power profile vector.

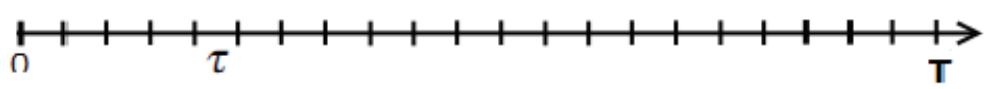

Fig. 6 Time horizon.

where either sum may be empty if $t_{s}^{i}=t_{1}$ or $t_{f}^{i}=t_{T}$. If both equalities hold (say if the user does not specify a comfort interval) the constraints vanish. To enforce that appliance $i$ runs $n_{i}$ times in $\left\{t_{s}^{i}, \ldots, t_{j}^{i}\right\}$, we need the following constraints,

$$
\forall i \quad \sum_{t \in\left\{t_{s}^{i}, \ldots, t_{f}^{i}\right\}} x_{j}^{i}(t)=n_{i}, \forall j: j \in J_{i}
$$

Phases or virtual appliances can be kept in order by imposing the following constraint,

$$
\forall i \quad \sum_{t \in \tau}\left[t \cdot x_{j+1}^{i}(t)-t \cdot x_{j}^{i}(t)\right] \geq 1, \forall j: j \in J_{i}
$$

and to prevent interruption between any two consecutive phases (virtual appliances), we use the following constraint, note that it is almost the same as the previous constraint but with " $=$ " replacing " $\geq "$

$$
\forall i \quad \sum_{t \in \tau}\left[t \cdot x_{j+1}^{i}(t)-t \cdot x_{j}^{i}(t)\right]=1, \forall j: j \in J_{i}
$$

If the user wants to make sure that one task is scheduled after another task (For example, Laundry dryer has to be scheduled after washing machine), then we need to use the following constraints,

$$
\begin{gathered}
\forall i \quad \sum_{0}^{T} t \cdot x_{j}^{i}(t)-\sum_{0}^{T} t \cdot x_{j}^{k}(t) \geq 0 \\
x_{j}^{i}(t)+x_{j}^{k}(t)<=0
\end{gathered}
$$

where, $k \in\{1,2, \ldots, m\}$ and $k \neq i$.

\subsection{Uniphase Appliances}

Some of household appliances are uniphase, $\dot{\mathcal{A}} \subset \mathcal{A}$. This kind of appliances can be considered as a special case of multiphase appliances. In order to reduce the complexity of the problem (by reducing the number of binary decision variables), a special MILP formulation will be used for uniphase appliances. Let us assume that a house has a set of uniphase appliances, $n=|\dot{\mathcal{A}}|$. The power profile of uni-phase appliances, shown in Fig. 3a, is split into a set of identical phases, called virtual appliance $\mathcal{J}_{i}$. Then, we can pick one phase and run it $\Delta_{i}$ times. In addition, the virtual appliances need an auxiliary binary variable $\delta_{i}(t)$.

$$
\forall i \quad P_{i}(t)=\alpha_{i} \cdot x_{i}(t) \in\left\{0, \ldots, \alpha_{\Delta_{i}}\right\} . \forall t: t \in \tau
$$

The following constraint is used to specify the potential operating time of a uni-phase appliance. In other words, users can specify the comfortable time for running their appliances.

$$
\forall i \sum_{t=0}^{t_{s}^{i}-1} x_{i}(t)+\sum_{t=t_{f 1}^{i}+1}^{t_{T}} x_{i}(t)=0
$$

Phases can be kept adjacent with the aid of the auxiliary binary variable $\delta_{i}(t)$.

$$
\begin{gathered}
\forall i \quad x_{i}(t)+\delta_{i}(t) \leq 1, \forall t: t \in \tau, \\
\forall i \quad \delta_{i}(t-1)-\delta_{i}(t) \leq 0, \forall t: t \in \tau \\
\forall i \quad x_{i}(t-1)-x_{i}(t)-\delta_{i}(t) \leq 0, \forall t: t \in \tau .
\end{gathered}
$$

The sequences or the order of virtual appliances (phases) is not necessary for uni-phase appliances because it does not make any difference concerning cost and task. Fig. 5a represents the power profile of uniphase appliances, whereas Fig. 5b represents the 
power profile of multiphase appliances.

\subsection{Plug-in Hybrid Electric Vehicle}

PHEV (plug-in hybrid electric vehicle) is not a household appliance but it consumes electricity from the house to recharge its battery. Therefore, we consider it as an appliance. Let us assume that the house has a set of PHEVs, $\mathcal{A}^{\prime} \subset \mathcal{A}$. The operation of some appliances depends on external conditions rather than initial user demands. For instance, charging a battery depends on the battery charging state $v_{i}(t)$ and its charging rate, $\alpha_{i}$, and its initial and final state of charge, $\underline{\Theta_{i}}$ and $\overline{\Theta_{i}}$ respectively, whereas the operation of an $\mathrm{AC}$ unit depends on the room temperature, $T_{i n}^{i}(t)$, the outside temperature and the appliance's heating or cooling power. In the case of batteries, we need to use Eqs. (17) and (18).

$$
\begin{gathered}
\forall i \quad v_{i}(t)=v_{i}(t-1)+\frac{1}{4} \cdot \pi \cdot P_{i}(t), \forall t: t \in\left\{t_{s}^{i}, \ldots, t_{f}^{i}\right\} \\
\forall i \quad v_{i}\left(t_{s}^{i}\right)=\underline{\Theta}_{i}, \text { and } v_{i}\left(t_{f}^{i}\right)=\bar{\Theta}_{i},
\end{gathered}
$$

where, $\underline{\Theta_{i}^{h}}$ is the initial state of charge of the battery,

$\overline{\Theta_{i}^{h}}$ is the desired final state of charge of the battery (usually full), and $\pi_{h, i}$ is the battery charging efficiency.

\subsection{Air Conditioning System}

Let us assume that the house has a set of $\mathrm{AC}$ units, $\hat{\mathcal{A}} \subset \mathcal{A}$. The main task of $\mathrm{AC}$ unit or electric heater is to keep the room temperature within the comfort level $\left[T_{\min }^{r}, T_{\max }^{r}\right]$ during $b_{i}^{r}$ specified time intervals $I_{1}^{r}, \ldots, I_{b_{i}}^{r}$. The relationship between room temperature and the power allocated to the appliance is shown in Eqs. (19) and (20).

$$
\begin{gathered}
\forall i \quad T_{\text {in }}^{r}(t)=\epsilon_{i} T_{\text {in }}^{r}(t-1) \\
+\left(1-\epsilon_{i}\right)\left[T_{\text {out }}(t)-\frac{\eta}{k} P_{i}^{r}(t)\right] \\
\forall i T_{\text {min }}^{i} \leq T_{\text {in }}^{i}(t) \leq T_{\text {max }}^{i} \forall t: t \in I_{1} \cup \ldots I_{b_{i}}
\end{gathered}
$$

where, $\epsilon_{i}$ is the appliance inertia, $\eta$ is the efficiency of the system (with $\eta>0$ for a heating appliance and $\eta$ $<0$ in the case of cooling), $k$ is the thermal conductivity, $T_{\text {out }}(t)$ is the outside temperature at time $t$.

The allocated power to HVAC system is a bit different, AC unit consume $\left\{\alpha_{1}, \alpha_{2}, \ldots, \alpha_{k_{c, i}^{r}}\right\}$ in case of cooling and $\left\{\beta_{1}, \beta_{2}, \ldots, \beta_{k_{h, i}^{r}}^{r}\right\}$ in case of heating. The $i$ th appliance in room $r$ can cool things down (heat things up) in $k_{c, i}^{r}$ (resp. $k_{h, i}^{r}$ ) different ways, then its power consumption at time $t$ can be defined as

$$
\begin{aligned}
\forall i P_{i}^{r}(t)=\sum_{j=1}^{k_{c, i}^{r}} \alpha_{i}^{r} & x_{i, j}^{r}(t) \\
& +\sum_{j=1}^{k_{h, i}^{r}} \beta_{i}^{r} y_{i, j}^{r}(t) \quad \forall t: t \in \mathcal{T}
\end{aligned}
$$

where

$$
\forall i, \forall j x_{i, j}^{r}(t), y_{i, j}^{r}(t) \in\{0,1\} \quad \forall t: t \in \mathcal{T}
$$

And

$$
\forall i \sum_{j=1}^{k_{c, i}^{r}} x_{i, j}^{r}(t)+\sum_{j=1}^{k_{h, i}^{r}} y_{i, j}^{r}(t) \leq 1 \quad \forall t: t \in \mathcal{T}
$$

The total power allocated in apartment $r$ at time $t$ is

$$
P_{r}(t)=\sum_{i=1}^{n_{r}} P_{i}^{r}(t) \forall t: t \in \mathcal{T}
$$

In addition, the possible allocated power to a set of identical $\mathrm{AC}$ units cool/heat in a room, $r$, could be $\Gamma=\left\{0, \alpha, 2 \times \alpha, \ldots, N_{r} \times \alpha\right\}$, the number of working modes is $N_{r}+1$, whereas, it is $2^{N_{r}}$ if the AC units are not identical.

\subsection{Objective Function and Additional Constraints}

For the purpose of our experiments, the general model (presented in Section 2.3) is simplified. The cost function $\Psi$ in Eq. (1) is replaced by the linear function, 


$$
\begin{gathered}
\Psi=\sum_{\mathrm{t} \in \mathcal{T}}\left\{\lambda(\mathrm{t}) \cdot \mathrm{L}_{\mathrm{g}}(\mathrm{t})+\xi(\mathrm{t}) \cdot \mathrm{r}(\mathrm{t})-\zeta(\mathrm{t})\right. \\
\cdot \mathrm{E}(\mathrm{t})\}
\end{gathered}
$$

and the discomfort function in Eq. (2) is replaced by the following linear function,

$$
\Omega=\sum_{r \in R} \sum_{t \in I_{j}^{r}}\left|T_{i n}^{r}(t)-T_{o p t}^{r}\right|
$$

The objective function is presented in the following Eq. (27):

$$
\operatorname{Min}(\Psi, \Omega)
$$

subject to all the constraints defined in this section as well as a few more involving functions $L_{g}, L_{r}$ and $E$. Thus, the exported renewable power to UG and the consumed renewable power at any time must be equal to the predicted renewable power,

$$
E(t)+L_{r}(t)=P_{\text {rew }}(t) \quad \forall t: t \in \mathcal{T}
$$

where, $P_{\text {rew }}(t)$ is the renewable power available at time $t$. The power allocated to the building at any time slot, $t$, must be equal to the building demand,

$$
\begin{aligned}
L_{g}(t)+L_{r}(t)= & \sum_{r \in R} P_{r}(t)+\sum_{i=1}^{n} P_{j}^{i}(t) \\
& +\sum_{i=1}^{m} P_{1}^{i}(t) \forall t: t \in \mathcal{T}
\end{aligned}
$$

\subsection{MILP Formulation Issues}

There are two issues with the objective function in Eq. (27). The first one is that, to the best knowledge of the authors, there is no LP solver that can tackle MILP-based MOOP (multi-objective optimization problem). Therefore, we need to convert the problem into an SOOP (single objective optimization problem). The second issue is that we cannot use an absolute variable directly in linear programming. Therefore, we need to represent Eq. (26) to eliminate the absolute sign. We can represent any function by introducing an auxiliary variable (eg. $\operatorname{Min}|f(x)|$, that can be represented as Min $\mathrm{Z}$ subject to $f(x) \leq \mathrm{Z}$ and $-f(x) \leq \mathrm{Z}$ [38]. So, to reformulate the absolute value, $\left|T_{i n}^{r}(t)-T_{o p t}^{r}\right|$, we will add another variable $\delta(t)$ and two more constraints in Eq. (30) to the problem and swap Eqs. (26) with Eqs. (31).

$-T_{i n}^{r}(t)+T_{o p t}^{r} \leq \delta(t)$ and $T_{i n}^{r}(t)-T_{o p t}^{r} \leq \delta(t)(30)$

$$
\Phi=\sum_{r \in R} \sum_{t \in I_{j}^{r}} \delta_{r}(t)
$$

having coped with the absolute sign issue, we will tackle the MOOP issue. A linear scalarization technique will be used to convert MOOP into SOOP, the objective function in Eq. (27) will be replaced by the following function,

$$
\operatorname{Min}\left(\omega_{1} \Psi+\omega_{2} \Phi\right)
$$

where, $\omega_{1}$ and $\omega_{2}$ are weights to bias the optimization toward either cost or comfort.

\subsection{Problem Complexity}

The framework presented so far leads to an implementation of an MILP-based algorithm for П. However, there is strong evidence suggesting that the problem may be rather difficult computationally. The results, in our previous paper [39], clearly suggest that the underlying LP solver speed is heavily affected by the resolution of time slots (the number of time slots) or the number of appliances in the building. Furthermore, the problem is in fact NP-hard [40] even if the building has a single apartment and a single $\mathrm{AC}$ unit that has $k$ working levels and $k>2$. Therefore, there is little hope that an exact algorithm can solve such problems, the outcomes of such analysis led us to the study of effective heuristic algorithms that can be used to obtain sub-optimal feasible solutions relatively quickly.

\section{Relaxation and Rounding}

We will use LP relaxation and rounding with interruptible appliances only. LP relaxation and rounding is a well-known approach to cope with the computational intractability of MILP formulation. The relaxation is achieved by omitting all constraints restricting the values of some variables to be integer 
numbers [41]. In the specific case of $\Pi$, this can be done by replacing all constraints described in Eq. (22) by

$$
0 \leq x_{i, j}^{r}(t) \leq 1 \text {, and } 0 \leq y_{i, j}^{r}(t) \leq 1 \text {. }
$$

Solving the resulting problem can be done effectively and will lead to a solution that will have a cost no larger than that of an optimal solution for the original problem. However, there is no guarantee for the allocated power of any appliance. For instance, in case of running $\mathrm{AC}$ in cooling mode, $\alpha$, will be equal to any of $\alpha_{1}, \ldots, \alpha_{k_{c, i}^{r}}^{r}$. Thus the resulting solution does not immediately translate into a schedule for the building's appliances (eg. if $x_{i, j}^{r}(5)=0.4785$, do we switch appliance $i$ "ON" at level $j$ or not?). Note that we cannot allocate $0.4785 \times \alpha_{j}$ to an $\mathrm{AC}$ unit, doing so could damage the $\mathrm{AC}$ unit or make it operate in an inefficient way. We now present a rounding strategy that can be used to get feasible solutions for П. Two of the algorithms compared in Section 5 are based on such a strategy.

MDR (minimum deviation rounding) works on the solution produced by the LP relaxation and generates (in polynomial time) a feasible solution for the initial MILP problem. Different apartments are treated independently. Let us assume that $\Gamma_{\mathrm{r}}=\left\{\alpha_{1}, \beta_{1}, \ldots, \alpha_{k_{c, i}^{r}}^{r}, \beta_{h, i}^{r}\right\} \quad$ is the set of all permissible power values for apartment $r$. The rationale behind the MDR algorithm is to round the allocated power to the first permissible power value, say $\widetilde{P}_{r}(\mathrm{t})=\alpha_{1}$, and calculate the deviation between $T_{i n}^{r}(t)$ and $\widetilde{T_{i n}^{r}}(\mathrm{t})$, where $\widetilde{T_{l n}^{r}}(\mathrm{t})$ is the room temperature in case we allocated $\alpha_{1}$ to the AC unit. Then, do the same with the rest. After that, we pick the best permissible power value that gives the smallest temperature deviation and proves it as solution as long as it does not violate other constraints. Eqs. (34) and (35) show how does MDR work in case of cooling. Note, in case of heating swap $\alpha$ with $\beta$.

$$
\Delta_{k}=\left|\widetilde{T_{l n}^{r, k}}(t)-T_{i n}^{r}(t)\right|
$$

$$
\begin{gathered}
\forall r, \forall t, \quad k=0,1, \ldots, K \\
\widetilde{P}_{r}(\mathrm{t})=\alpha_{\mathrm{m}}, \quad \text { and } \Delta_{\mathrm{m}} \leq \Delta_{\mathrm{j}}, \\
\mathrm{m} \neq \mathrm{j}, \mathrm{j}, \quad \mathrm{m}=0,1, \ldots, K
\end{gathered}
$$

where, $\widetilde{T_{l n}^{r, k}}(\mathrm{t})$ is the $r$ th apartment temperature when $\widetilde{P}_{r}(\mathrm{t})=\alpha_{k}$. Pseudo code of MDR is shown in Algorithm 1 and 2. MCR strategy is designed for uni-phase appliances, $i \in \dot{\mathcal{A}}$. It rounds the value that gives the lowest cost among a set of candidate values. For example, Let us assume that we need to run appliance ( $\alpha=3.5 \mathrm{kWh}$ ) for one hour. Let us assume that the time horizon is 5 hours, and each time slot is $\tau$ $=1$ hour, and the electricity price and generated power is shown in Fig. 7. It is clear that running the appliance in the first and the last time slot would not be optimal because there is no renewable power. Assume that after using LP relaxation, the optimizer allocated $28 \%$ of $\alpha$ in $\tau=2,44 \%$ of $\alpha$ in $\tau=3$, and $28 \%$ of $\alpha$ in $\tau=3$. Practically, this is not possible. Therefore, this allocated power must be rounded to either 0 or $\alpha=3.5 \mathrm{~kW}$. We will consider all the costs and pick the cheapest one, which is in this case at time slot 2. Note, we have tried couple of strategies to round the allocated power such as rounding the allocated power at off-peak electricity price ( $\tau=4$ and $\tau=5)$. Also, rounding the allocated power where there is highest surplus renewable power left $(\tau=3)$.

\section{Results and Discussions}

The experiments in this paper have been done on a PC with an Intel(R) Core(TM) i7-2600 CPU @ 3.4 GHZ, RAM is 16 GB, 64-bit Operating System (Windows 7). Also, Gurobi has been used to solve LP and MILP problems, whereas Java was the main tool to build the proposed mathematical model. In the rest of this section we will compare two different heuristic ways of finding feasible solutions for instances of $\Pi$ : using a truncated version of the MILP (MILP-H), using MDR algorithm, or a slightly faster version of the same process, named MDR V, which omits step 9 in procedure MDR. Three case studies will be 


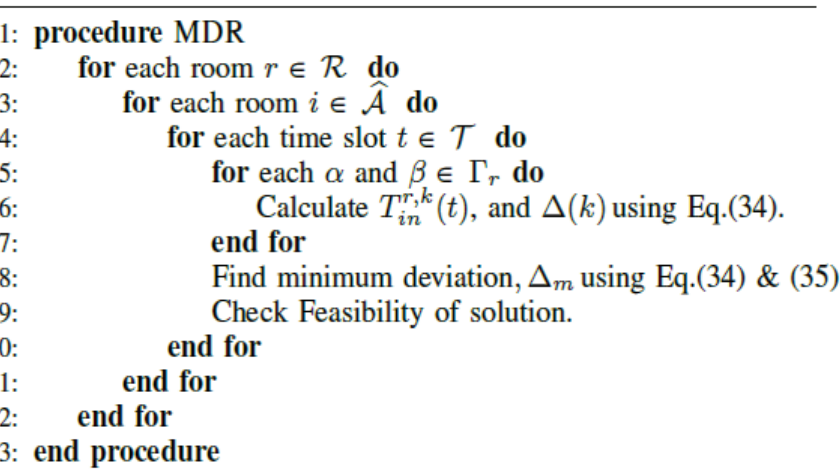

Algorithm 1 Minimum deviation rounding (MDR).

$$
\begin{aligned}
& \text { 1: } \text { procedure Check FeAsibility } \\
& \text { 2: } \quad \text { for each time slot, } t \in I_{1}^{r} \cup, \ldots, I_{b_{i}^{r}}^{r} \text { do } \\
& \text { 3: } \quad \text { Calculate } \widetilde{T_{i n}^{r}}(t) \text { using } \widetilde{P_{r}}(t) \leftarrow \alpha_{m} \text { or } \beta_{m} . \\
& \text { 4: } \quad \text { if } \widetilde{T_{i n}^{r}}(t)>T_{m a x} \text { then } \\
& \text { 5: } \quad \text { Adjust } \widetilde{P_{r}}(t), \widetilde{P_{r}}(t) \leftarrow \alpha_{m+1} \text { or } \beta_{m+1} . \\
& \text { 6: } \quad \text { end if } \\
& \text { 7: } \quad \text { if } \widetilde{T_{i n}^{r}}(t)<T_{\min } \text { then } \\
& \text { 8: } \quad \text { Adjust } \widetilde{P_{r}}(t), \widetilde{P_{r}}(t) \leftarrow \alpha_{m-1} \text { or } \beta_{m-1} . \\
& \text { 9: } \quad \text { end if } \\
& \text { end for } \\
& \text { Check that MDR does not violate (19) } \\
& \text { Update All dependent variables. }
\end{aligned}
$$

Algorithm 2 Checking feasibility of MDR's solution.

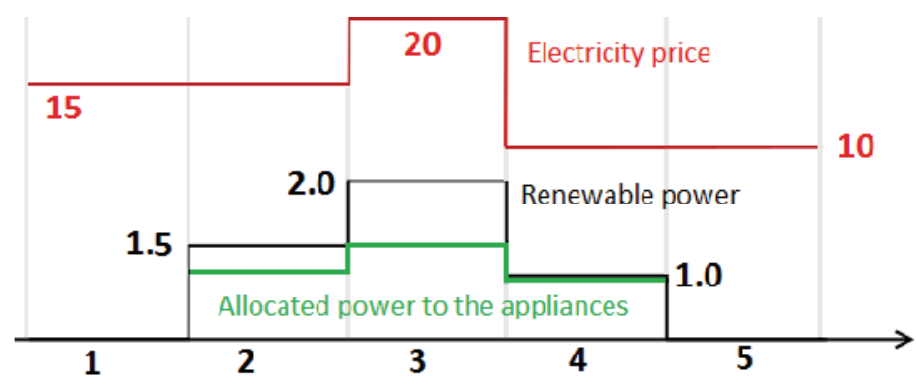

Fig. 7 Time horizon.

demonstrated in these results to compare the heuristic algorithms.

\subsection{Communal Input Setting}

All case studies will use the same input data in this section. All AC units have the same inertia, $\forall i, \epsilon_{i}=0.96 ; \forall r, k_{r}=0.98 \quad \mathrm{KW} /{ }^{\circ} \mathrm{C}$. Also, all scenarios will use $T_{\min }^{r}=18.0{ }^{\circ} \mathrm{C}, T_{\max }^{r}=22.0{ }^{\circ} \mathrm{C}$ and $T_{o p t}^{r}=20.0{ }^{\circ} \mathrm{C}$. Locally generated renewable energy costs nothing ( $\xi=0.0 \mathrm{p} / \mathrm{kWh})$, and the building benefits of an export tariff $\zeta=5.0 \mathrm{p} / \mathrm{kWh} . \omega_{1}=\omega_{2}=$ 1. The first chart in Fig. 8 shows two pricing schemes, a "Fixed" and "Dynamic" pricing. The second chart illustrates predicted renewable power in three days, a completely cloudy day $(0.0 \mathrm{~kW})$, a partly cloudy, and a sunny day (blue sky). The last chart in the same figure demonstrates the outside temperature.

\subsection{The First Case Study}

The primary aim of this case study is to illustrate how appropriate the proposed heuristic algorithm in terms of cost and runtime compared with the exact MILP-based optimization algorithm.

(1) Input settings: Let us assume that we have a 
small building (two bedrooms house, Fig. 9), the house has a set of electric household appliances, see Table 1. We need to keep the room temperature in comfortable level. The number of rooms is $R=3$ including the living room. The number of AC units $N$ $=6$ identical $\mathrm{AC}$ units. Furthermore, the living room, $r$ $=1$, has 3 identical $\mathrm{AC}$ units, $N_{1}=3 \mathrm{AC}$ units, the master bedroom, $r=2$, has two identical AC units, $N_{2}$ $=2 \mathrm{AC}$ units, and the second bedroom, $r=3$, has one AC unit. The possible allocated power to the living room, master bedroom and second bedroom is $\Lambda_{1}=\{0,2.3,4.6,6.9\} \quad, \quad \Lambda_{2}=\{0,2.3,4.6\}, \quad$ and $\Lambda_{3}=\{0,2.3\}$, respectively. $\epsilon_{1}=\epsilon_{2}=\epsilon_{3}=0.96$, $\eta_{1}=10, \eta_{2}=20, \eta_{3}=30$, respectively. $k_{1}=k_{2}=$ $k_{3}=0.98 \mathrm{KW} /{ }^{\circ} \mathrm{C}, \xi=0.0 \$ / \mathrm{kWh}, \zeta=0.05 \$ / \mathrm{kWh}$. Two pricing schemes will be used in this case study, which are Fixed and Dynamic pricing schemes, see the first chart in Fig. 8. Also, this flat is provided with domestic renewable resources, PV arrays. These PV arrays generate a maximum amount of $4.1 \mathrm{kWh}$ of solar power, three shapes of renewable power are used, zero (cloudy day), bell shape (sunny day), and intermittent form (partly cloudy day), see the second chart in Fig. 8. The time horizon is split into $T=288$ time slots, $\tau=5 \mathrm{~min}$. $T_{\min }^{r}=18.0{ }^{\circ} \mathrm{C}$ and $T_{\max }^{r}=$ $22.0^{\circ} \mathrm{C}$ for all $r$ in two periods of the day, $b_{r}=2$, see Table 2 for more details. Table 5 and 6 show the power profile of multiphase and uniphase appliances, respectively, that will be used in this paper. Two scenarios will be used in this case study to demonstrate the performance of the proposed heuristic algorithm with two different pricing strategies. We will use just the data of one day (Sunny day).

(2) Findings: Table 3 compares between using exact and heuristic algorithm. Table 4 demonstrates that solving the problem at hand to optimality is not feasible by using the current MILP solvers (solving the problem to optimality when $\tau=1$ minute took more than 13 hours). Also, the table reveals how good using heuristic algorithm to solve NP-hard optimization problems. Furthermore, Fig. 10 illustrates an exact and a heuristic solution of the problems, it shows details of power allocation of each appliances in the flat.

\subsection{The Second Case Study}

The primary intention of this case study is to do a scalability test of the proposed heuristic algorithm. In other words, the main objective is to investigate the performance of the proposed heuristic algorithm regarding the runtime especially when the size of the computational problem is massive (a large number of household appliances and high time resolution ( $\tau$ is small)). In addition, we will compare the sub-optimal solution provided by the heuristic algorithm using LPR (LP relaxation) and rounding techniques with the sub-optimal solution provided by built-in MILP-H (heuristic MILP).

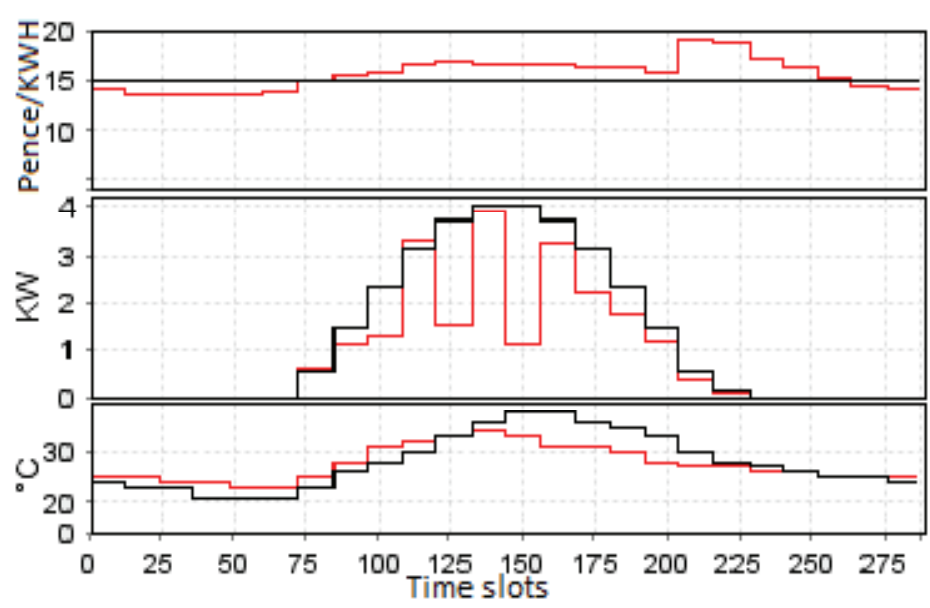

Fig. 8 First chart shows electricity prices, second one shows the predicted renewable power, day 2 in red and day 3 in black, and the last one shows the outside temperature, day 1 and day 2 in red and day 3 in black. 


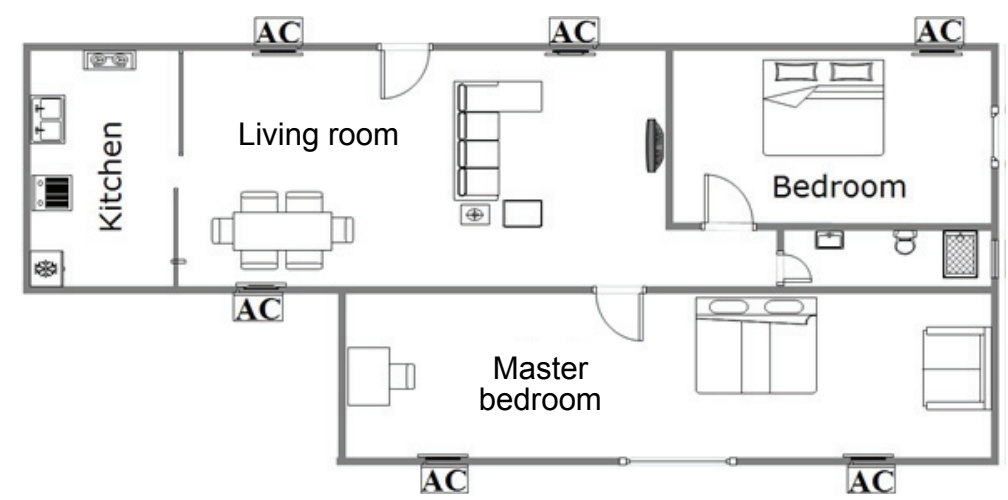

Fig. 9 Residential building.

Table 1 Electric appliances.

\begin{tabular}{llll}
\hline Appliance & No. & Inuterruptible & Multiphase \\
\hline Water heater & 1 & Yes & No \\
Towel radiator & 1 & Yes & No \\
PHEV & 1 & Yes & No \\
Washing machine & 1 & No & Yes \\
Dishwasher & 1 & No & Yes \\
Laundry dryer & 1 & No & Yes \\
AC unit & 6 & Yes & No \\
Electric cooker & 1 & No & No \\
\hline
\end{tabular}

Table 2 Comfort time.

\begin{tabular}{lll}
\hline Appliance & Start time & End time \\
\hline Water heater & $01: 00$ & $07: 00$ \\
Towel radiator & $01: 00$ & $17: 00$ \\
PHEV & $18: 00$ & $07: 00$ \\
Washing machine & $23: 00$ & $12: 00$ \\
Dishwasher & $20: 00$ & $07: 00$ \\
Laundry dryer & $01: 00$ & $13: 00$ \\
AC unit & $05: 00,14: 00$ & $12: 00,23: 00$ \\
Electric cooker & $18: 00$ & $20: 00$ \\
\hline
\end{tabular}

Table 3 Runtime and operating cost.

\begin{tabular}{|c|c|c|c|c|c|c|c|c|c|c|c|c|c|}
\hline \multirow[b]{2}{*}{ Price } & \multirow[b]{2}{*}{$\tau$ in $\min$} & \multicolumn{6}{|c|}{ Exact algorithm (MILP) } & \multicolumn{6}{|c|}{ Heuristic algorithm } \\
\hline & & 30 & 20 & 15 & 10 & 5 & 1 & 30 & 20 & 15 & 10 & 5 & 1 \\
\hline \multirow{2}{*}{ Dynamic } & Runtime (sec.) & 27 & 98 & 1,463 & 3,145 & 35,600 & 46,800 & 21 & 34 & 42 & 83 & 123 & 421 \\
\hline & Cost $(\$)$ & 2.28 & 2.11 & 2.07 & 2.03 & 1.99 & 1.97 & 2.53 & 2.31 & 2.14 & 2.09 & 2.03 & 2.01 \\
\hline \multirow{2}{*}{ Fixed } & Runtime (sec.) & 9 & 51 & 984 & 2,331 & 4,680 & 32,400 & 8 & 27 & 33 & 51 & 97 & 332 \\
\hline & Cost $(\$)$ & 2.54 & 2.32 & 2.19 & 2.11 & 2.09 & 2.08 & 2.71 & 2.55 & 2.38 & 2.29 & 2.18 & 2.19 \\
\hline
\end{tabular}

Table 4 The optimal cost and the maximum saving in $\$$.

\begin{tabular}{llllll}
\hline & Pricing strategies & Maximum cost (\$) & Minimum cost (\$) & Runtime & Maximum saving (\%) \\
\hline \multirow{2}{*}{ Day 1 } & Fixed pricing & 3.881 & 3.881 & $76 \mathrm{~s}$ & 00.00 \\
& Dynamic pricing & 5.243 & 4.232 & $147 \mathrm{~s}$ & 19.14 \\
\multirow{2}{*}{ Day 2 } & Fixed pricing & 3.167 & 1.504 & $4 \mathrm{~h}, 34 \mathrm{~min}$ & 52.59 \\
& Dynamic pricing & 3.663 & 1.691 & $19 \mathrm{~h}, 47 \mathrm{~min}$ & 53.80 \\
Day 3 & Fixed pricing & 3.167 & 1.101 & $13 \mathrm{~h}, 22 \mathrm{~min}$ & 65.20 \\
& Dynamic pricing & 3.736 & 1.191 & $27 \mathrm{~h}, 02 \mathrm{~min}$ & 68.17 \\
\hline
\end{tabular}


Table 5 Multiphase uninterruptible appliances, all time unit in minutes and all power unit in $\mathrm{kW}$.

\begin{tabular}{lllllll}
\hline Laundry dryer & $\alpha$ & 3.2 & 0.28 & 0 & 3.2 & 0.28 \\
& $\Delta_{\min }$ & 15 & 10 & 5 & 20 & 10 \\
Dishwasher & $\alpha$ & 0.2 & 2.7 & 0.2 & 2.7 & 0.2 \\
& $\Delta_{\min }$ & 5 & 15 & 15 & 20 & 5 \\
\multirow{2}{*}{ Washing machine } & $\alpha$ & 2.2 & 0.28 & 2.2 & 0.28 & - \\
& $\Delta_{\min }$ & 10 & 20 & 10 & 20 & - \\
\hline
\end{tabular}

Table 6 Interruptible appliances.

\begin{tabular}{lll}
\hline Appliances & $\alpha$ & Depend on \\
\hline Water heater & $3.1 \mathrm{~kW} / \mathrm{t}$ & - \\
Towel radiator & $1.5 \mathrm{~kW} / \mathrm{t}$ & - \\
Electric cooker & $2.5 \mathrm{~kW} / \mathrm{t}$ & - \\
PHEV & $0.35 \mathrm{~kW} / \mathrm{t}$ & $\Theta\left(t_{s}\right)=2.0, \Theta\left(t_{f}\right)=16.0$ \\
Air conditioner & $2.3 \mathrm{~kW} / \mathrm{t}$ & $T_{\min }=18, T_{\max }=22$ \\
\hline
\end{tabular}

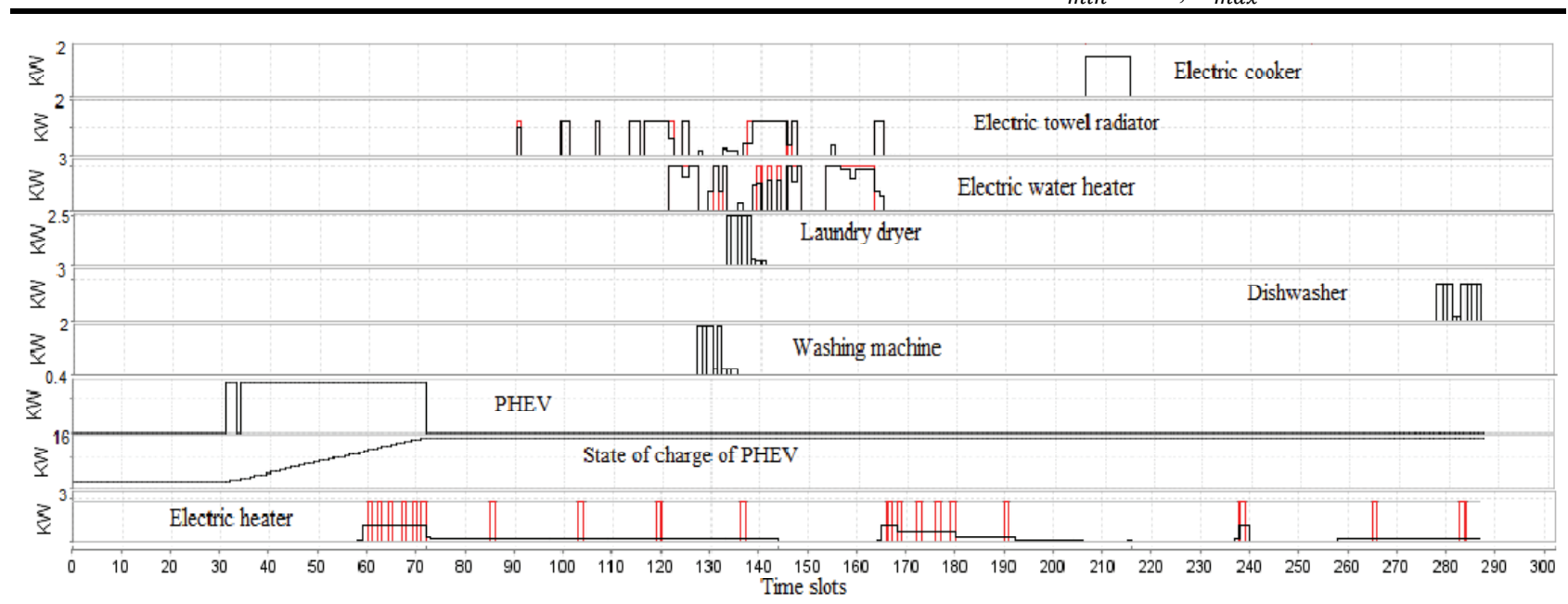

Fig. 10 Power allocation.

(1) Input settings: As the AC units or heaters are the most complicated appliances and add a lot of complicity to the model (more than other appliances such as dishwasher or washing machine). We will use just AC units in these experiments. We will vary the number of appliances (AC units) from 1 appliance to 300 appliances in the building. In addition, we will vary the time resolution from 30 minutes to 1 minute. Note, the complexity of the problem is a function of time resolution and the number of appliances.

(2) Results: Fig. 12 demonstrates that the exact algorithm (MILP) cannot solve large problems in polynomial time as the problem at hand is an NP-hard. On the other side, Fig. 11 reveals that the proposed heuristic algorithm (LPR) can solve the problem to sub-optimality in polynomial time. Furthermore,
Table 7 illustrates that in a small problem the built-in MILP-Heuristic algorithm (MILP-H) gives a better solution than our heuristic algorithm (LPR), whereas in large problems, the proposed heuristic algorithm (LPR) gives a better solution compared with MILP-H.

\section{Conclusions}

To conclude, this work illustrates the feasibility of using LP relaxation and rounding technique as a heuristic optimization algorithm of power management problems. The proposed algorithm can be used for solving huge optimization problems, the results show that the sub-optimal cost of a large building working within a smart grid is cheaper than the sub-optimal cost in a large problem using built-in MILP-H. 


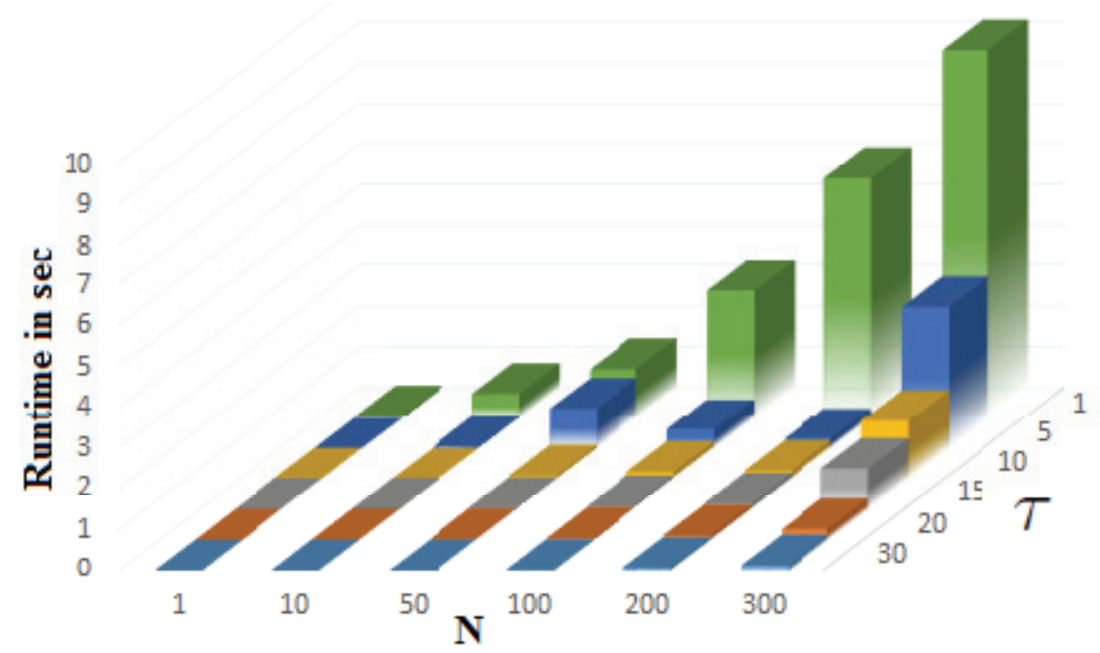

Fig. 11 Runtime of LPR in seconds.

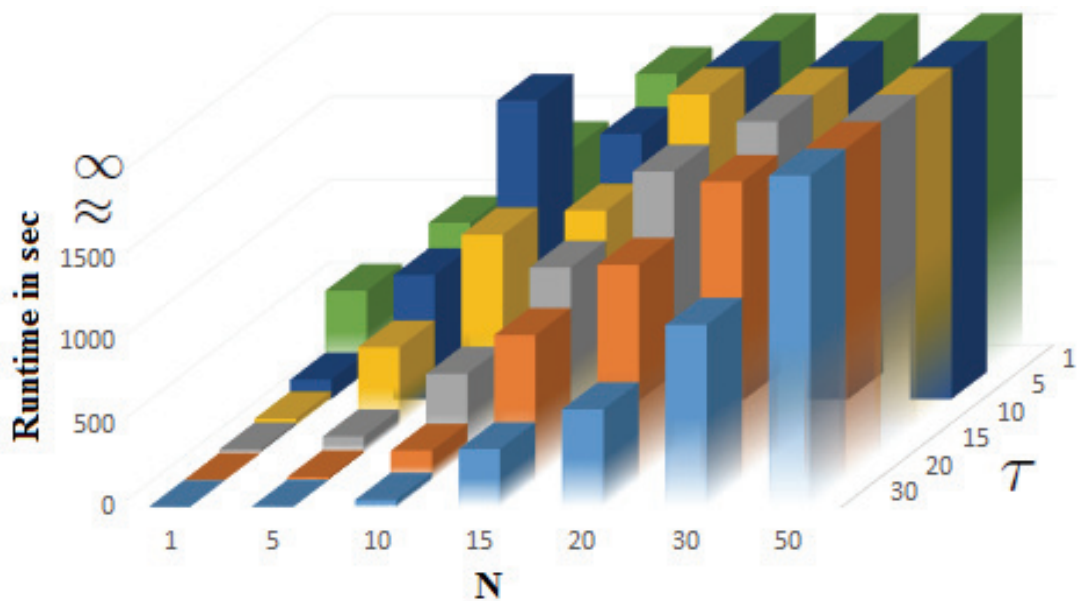

Fig. 12 Runtime of MILP in seconds.

Table 7 Comparison between our heuristic algorithm (LPR) and built-in heuristic algorithm (MILP-H).

\begin{tabular}{lllllll}
\hline \multicolumn{1}{c}{$N \backslash \tau$} & 30 & 20 & 15 & 10 & 5 & 1 \\
\hline 1 & MILP-H & MILP-H & MILP-H & MILP-H & MILP-H & MILP-H \\
10 & MILP-H & MILP-H & MILP-H & MILP-H & $=$ & LPR \\
50 & MILP-H & MILP-H & MILP-H & MILP-H & LPR & LPR \\
100 & MILP-H & MILP-H & $=$ & LPR & LPR & LPR \\
200 & MILP-H & $=$ & LPR & LPR & LPR & LPR \\
300 & MILP-H & LPR & LPR & LPR & LPR & LPR \\
\hline
\end{tabular}

\section{References}

[1] Rosa, A. V. D. 2005. Fundamentals of Renewable Energy Processes. Academic Press.

[2] Aman, S., Simmhan, Y., and Prasanna, V. K. 2013. "Energy Management Systems: State of the Art and Emerging Trends." IEEE Communications Magazine 51 (1): 114-9.

[3] Momoh, J. 2012. Smart Grid: Fundamentals of Design and Analysis. Vol. 63. John Wiley \& Sons.
[4] Chai, B., Chen, J., Yang, Z., and Zhang, Y. 2014. "Demand Response Management with Multiple Utility Companies: A Two-Level Game Approach." IEEE Transactions on Smart Grid 5 (2): 722-31.

[5] Mohsenian-Rad, A.-H., Wong, V. W. S., Jatskevich, J., Schober, R., and Leon-Garcia, A. 2010. "Autonomous Demand-Side Management Based on Game-Theoretic Energy Consumption Scheduling for the Future Smart Grid." IEEE Transactions on Smart Grid 1 (3): 320-31.

[6] Matallanas, E., Castillo-Cagigal, M., Gutiérrez, A., 
Monasterio-Huelin, F., Caamaño-Martín, E., Masa, D., and Jiménez-Leube, J. 2012. "Neural Network Controller for Active Demand-Side Management with PV Energy in the Residential Sector." Applied Energy 91 (1): 90-7.

[7] Argiriou, A. A., Bellas-Velidis, I., and Balaras, C. A. 2000. "Development of a Neural Network Heating Controller for Solar Buildings." Neural Networks 13 (7): 811-20.

[8] Mangiatordi, F., Pallotti, E., Vecchio, P. D., and Leccese, F. 2012. "Power Consumption Scheduling for Residential Buildings." In Proceedings of the 11th IEEE International Conference on Environment and Electrical Engineering (EEEIC), 926-30.

[9] Sepulveda, A., Paull, L., Morsi, W. G., Li, H., Diduch, C. P., and Chang, L. 2010. "A Novel Demand Side Management Program Using Water Heaters and Particle Swarm Optimization." In Proceedings of the IEEE Electric Power and Energy Conference (EPEC), 1-5.

[10] Gudi, N., Wang, L. and Devabhaktuni, V. et al. 2010. "Demand Response Simulation Implementing Heuristic Optimization for Home Energy Management." In North American Power Symposium (NAPS), 1-6.

[11] Gudi, N., Wang, L., and Devabhaktuni, V. et al. 2011. "A Demand-Side Management Simulation Platform Incorporating Optimal Management of Distributed Renewable Resources." In Power Systems Conference and Exposition (PSCE), 1-7.

[12] Gudi, N., Wang, L., and Devabhaktuni, V. 2012. "A Demand-Side Based Simulation Platform Incorporating Heuristic Optimization for Management of Household Appliances." International Journal of Electrical Power \& Energy Systems 43 (1): 185-93.

[13] Pedrasa, M., Spooner, T. D., and MacGill, I. F. 2009. "Scheduling of Demand Side Resources Using Binary Particle Swarm Optimization." IEEE Transactions on Power Systems 24 (3): 1173-81.

[14] Logenthiran, T., Srinivasan, D., and Shun, T. Z. 2012. "Demand Side Management in Smart Grid Using Heuristic Optimization." IEEE Transactions on Smart Grid 3 (3): 1244-52.

[15] Zhou, S., Zhang, X.-P., and Yang, X. 2012. "Design of Demand Management System for Household Heating Amp; Cooling." In Proceedings of the 3rd IEEE PES International Conference and Exhibition on Innovative Smart Grid Technologies (ISGT Europe), 1-6.

[16] Fernandes, F., Sousa, T., Silva, M., Morais, H., Vale, Z., and Faria, P. 2011. "Genetic Algorithm Methodology Applied to Intelligent House Control." In Proceedings of the IEEE Symposium on Computational Intelligence Applications in Smart Grid (CIASG), 1-8.

[17] Barbato, A., and Carpentieri, G. 2012. "Model and Algorithms for the Real Time Management of Residential
Electricity Demand." In Proceedings of the IEEE International Energy Conference and Exhibition (ENERGYCON), 701-6.

[18] Beaudin, M., Zareipour, H., and Schellenberg, A. 2012. "Residential Energy Management Using a Moving Window Algorithm." In Proceedings of the 3rd IEEE PES International Conference and Exhibition on Innovative Smart Grid Technologies (ISGT Europe), 1-8.

[19] Hubert, T., and Grijalva, S. 2012. "Modeling for Residential Electricity Optimization in Dynamic Pricing Environments." IEEE Transactions on Smart Grid 3 (4): 2224-31.

[20] Hao, H., Wu, D., Lian, J., and Yang, T. 2017. "Optimal Coordination of Building Loads and Energy Storage for Power Grid and End User Services." IEEE Transactions on Smart Grid PP (99): 1-1.

[21] Chen, Z., Wu, L., and Fu, Y. 2012. "Real Time Price Based Demand Response Management for Residential Appliances via Stochastic Optimization and Robust Optimization." IEEE Transactions on Smart Grid 3 (4): 1822-31.

[22] Chen, Y.-W., Chen, X., and Maxemchuk, N. 2012. "The Fair Allocation of Power to Air Conditioners on a Smart Grid." IEEE Transactions on Smart Grid 3 (4): 2188-95.

[23] Bozchalui, M. C., Hashmi, S. A., Hassen, H., Cañizares, C. A., and Bhattacharya, K. 2012. "Optimal Operation of Residential Energy Hubs in Smart Grids." IEEE Transactions on Smart Grid 3 (4): 1755-66.

[24] Brahman, F., Honarmand, M., and Jadid, S. 2015. "Optimal Electrical and Thermal Energy Management of a Residential Energy Hub, Integrating Demand Response and Energy Storage System." Energy and Buildings 90 (1): 65-75.

[25] Zong, Y., Mihet-Popa, L., Kullmann, D., Thavlov, A., Gehrke, O., and Bindner, H. W. 2012. "Model Predictive Controller for Active Demand Side Management with PV Self Consumption in an Intelligent Building." In Proceedings of the 3rd IEEE PES International Conference and Exhibition on Innovative Smart Grid Technologies (ISGT Europe), 1-8.

[26] Tsui, K. M., and Chan, S. C. 2012. "Demand Response Optimization for Smart Home Scheduling under Real-Time Pricing." IEEE Transactions on Smart Grid 3 (4): 1812-21.

[27] Barbato, A., Capone, A., Carello, M., Delfanti, G., Merlo, M., and Zaminga, A. 2011. "Cooperative and Non-cooperative House Energy Optimization in a Smart Grid Perspective." In IEEE International Symposium on a World of Wireless, Mobile and Multimedia Networks (WoWMoM), 1-6.

[28] Barbato, A., Capone, A., Carello, M., Delfanti, G., Merlo, M., and Zaminga, A. 2011. "House Energy Demand 
Optimization in Single and Multi-user Scenarios." In Proceedings of the IEEE International Conference on Smart Grid Communications (SmartGridComm), 345-50.

[29] Beaudin, M., Zareipour, H., and Schellenberg, A. 2012. "Residential Energy Management Using a Moving Window Algorithm." In Proceedings of the 3rd IEEE PES International Conference and Exhibition on Innovative Smart Grid Technologies (ISGT Europe), 1-8.

[30] Zong, Y., Kullmann, D., Thavlov, A., Gehrke, O., and Bindner, H. W. 2011. "Active Load Management in an Intelligent Building Using Model Predictive Control Strategy." In Proceedings of the IEEE Trondheim PowerTech, 1-6.

[31] Hubert, T., and Grijalva, S. 2011. "Realizing Smart Grid Benefits Requires Energy Optimization Algorithms at Residential Level." In Proceedings of the IEEE PES Innovative Smart Grid Technologies (ISGT), 1-8.

[32] Li, N., Chen, L., and Low, S. H. 2011. "Optimal Demand Response Based on Utility Maximization in Power Networks." In Proceedings of the IEEE Power and Energy Society General Meeting, 1-8.

[33] Mohsenian-Rad, A.-H., and Leon-Garcia, A. 2010. "Optimal Residential Load Control with Price Prediction in Real-Time Electricity Pricing Environments." IEEE Transactions on Smart Grid 1 (2): 120-33.

[34] Frangioni, A., Gentile, C., and Lacalandra, F. 2008. "Solving Unit Commitment Problems with General Ramp Constraints." International Journal of Electrical Power \&
Energy Systems 30 (5): 316-26.

[35] Kim, S.-J., and Giannakis, G. B. 2011. "Efficient and Scalable Demand Response for the Smart Power Grid." In Proceedings of the 4th IEEE International Workshop on Computational Advances in Multi-sensor Adaptive Processing, 109-12.

[36] Chen, X., Wei, T., and Hu, S. 2013. "Uncertainty-Aware Household Appliance Scheduling Considering Dynamic Electricity Pricing in Smart Home." IEEE Transactions on Smart Grid 4 (2): 932-41.

[37] Chai, B., Yang, Z., and Chen, J. 2013. "Optimal Residential Load Scheduling in Smart Grid: A Comprehensive Approach." In Proceedings of the 9th Asian Control Conference (ASCC), 1-6.

[38] Granger, B., Yu, M., and Zhou, K. 2014. "Optimization with Absolute Values." Accessed November 8, 2016. https://optimization.mccormick.northwestern.edu/index.p hp/Optimization_with_absolute_values.

[39] Arikiez, M., Grasso, F., and Zito, M. 2015. "Heuristics for the Cost-Effective Management of a Temperature Controlled Environment." In Proceedings of the IEEE Innovative Smart Grid Technologies-Asia (ISGT ASIA), $1-6$.

[40] Karaboghossian, T., and Zito, M. 2016. "Complexity of Renewable Energy Allocation Problems." Submitted for publication.

[41] Winston, W. L. 1995. Introduction to Mathematical Programming: Applications and Algorithms. 2nd Edition. International Thomson Publishing. 